Stud. Univ. Babeş-Bolyai Math. 62(2017), No. 2, 197-204

DOI: $10.24193 /$ subbmath.2017.2.06

\title{
Schwarzian derivative and Janowski convexity
}

\author{
Nisha Bohra and V. Ravichandran
}

\begin{abstract}
Sufficient conditions relating the Schwarzian derivative to the Janowski convexity of a normalized analytic function $f$ are obtained. As a consequence, sufficient conditions are determined for the function $f$ to be Janowski convex and convex of order $\alpha$. Also, some equivalent sharp inequalities are proved for $f$ to be Janowski convex.
\end{abstract}

Mathematics Subject Classification (2010): 30C45, 30C80.

Keywords: Schwarzian derivative, Janowski convexity, subordination.

\section{Introduction and main results}

Let $\mathcal{A}$ be the class of analytic functions $f$ in the open unit disk

$$
\mathbb{D}=\{z \in \mathbb{C}:|z|<1\}
$$

and normalized by the conditions $f(0)=0$ and $f^{\prime}(0)=1$. Let $\mathcal{S}$ be the class of univalent functions in $\mathcal{A}$. An analytic function $f$ is subordinate to an analytic function $g$, written as $f(z) \prec g(z)$, provided there is an analytic function $w$ defined on $\mathbb{D}$ with $w(0)=0$ and $|w(z)|<1$ satisfying $f(z)=g(w(z))$. For $-1 \leq B<A \leq 1$, let $\mathcal{P}[A, B]$ be the class consisting of normalized analytic functions $p(z)=1+c_{1} z+\cdots$ in $\mathbb{D}$ satisfying

$$
p(z) \prec \frac{1+A z}{1+B z} .
$$

The class $K[A, B]$ of Janowski convex functions [2] consists of functions $f \in \mathcal{A}$ satisfying

$$
1+\frac{z f^{\prime \prime}(z)}{f^{\prime}(z)} \in \mathcal{P}[A, B] .
$$

For $0 \leq \alpha<1, K[1-2 \alpha,-1] \equiv K(\alpha)$ is the usual class of convex functions of order $\alpha$. For $f \in \mathcal{S}$, the Schwarzian derivative of $f$ is defined as

$$
S_{f}(z)=\left(\frac{f^{\prime \prime}(z)}{f^{\prime}(z)}\right)^{\prime}-\frac{1}{2}\left(\frac{f^{\prime \prime}(z)}{f^{\prime}(z)}\right)^{2} .
$$


The Schwarzian derivative has the property that it is invariant with respect to Möbius transformations, that is, $S(M o f, z) \equiv S(f, z)$ for any Mobius transformation $M(z)$, and $S(M, z) \equiv 0$ if and only if $M(z)$ is a Möbius transformation. There are several sufficient conditions relating the Schwarzian derivative to the univalency of $f$ (see [5] and [6]). Miller and Mocanu in [3] determined sufficient conditions relating the Schwarzian derivative to the convexity of $f$. In this paper, we find the sufficient conditions for Janowski convexity of $f$. Also, Harmelin in [1] derived sharp bounds for $\left|(1-|z|)^{2} f^{\prime \prime}(z) / f^{\prime}(z)-2 \bar{z}\right|$ and for $\left(1-|z|^{2}\right)^{2}\left|S_{f}(z)\right|$, obtaining the refinement of Nehari's result [7] for convex functions of order $\alpha$. Here, we further extend this result for the class $K[A, B]$ of Janowski convex functions. Our first result gives a general condition for a function to be Janowski convex.

Theorem 1.1. Let $\Phi: \mathbb{C}^{2} \rightarrow \mathbb{C}$ satisfy $\operatorname{Re} \Phi\left(\frac{(1+A) \rho i+(1-A)}{(1+B) \rho i+(1-B)}, \tau+i \eta\right) \leq 0$ when $\rho, \tau, \eta \in \mathbb{R}$ and

$$
\begin{aligned}
& 2 \tau\left(\rho^{2}(1+B)^{2}+(1-B)^{2}\right)^{2}+\left(A^{2}-B^{2}\right)\left(\rho^{2}(1+B)-(1-B)\right)^{2} \\
& -4 \rho^{2}\left(A^{2}-B^{2}\right) \leq 0 .
\end{aligned}
$$

Let $f \in \mathcal{A}$ with $f^{\prime}(z) \neq 0$ and $(A-B) f^{\prime}(z)-(1+B) z f^{\prime \prime}(z) \neq 0$. If

$$
\operatorname{Re} \Phi\left(1+\frac{z f^{\prime \prime}(z)}{f^{\prime}(z)}, z^{2} S_{f}(z)\right)>0
$$

where $z \in \mathbb{D}$, then $f \in K[A, B]$.

Remark 1.2. For $A=1$ and $B=-1$, Theorem 1.1 reduces to [4, Theorem 4.6 b.].

Remark 1.3. The following functions satisfies the condition (1.1) of Theorem 1.1.

(1) $\Phi_{1}(u, v)=(A+B)(u-1)^{2}+2(A-B) v$,

(2) $\Phi_{2}(u, v)=2(A-B) v-(A+B)(\operatorname{Im} u)^{2}$.

Thus, we have the following.

Corollary 1.4. Let $f \in \mathcal{A}$ with $f^{\prime}(z) \neq 0$ and $(A-B) f^{\prime}(z)-(1+B) z f^{\prime \prime}(z) \neq 0$. Then each of the following is a sufficient condition for $f$ to be in $K[A, B]$.

(1) $\operatorname{Re}\left((A+B)\left(\frac{z f^{\prime \prime}(z)}{f^{\prime}(z)}\right)^{2}+2(A-B) z^{2} S_{f}(z)\right)>0$,

(2) $\operatorname{Re}\left(2(A-B) z^{2} S_{f}(z)-(A+B)\left(\operatorname{Im}\left(1+\frac{z f^{\prime \prime}(z)}{f^{\prime}(z)}\right)\right)^{2}\right)>0$.

For $A=1-2 \alpha, B=-1$, Theorem 1.1 gives the following sufficient condition for a function $f \in \mathcal{A}$ to be convex of order $\alpha$.

Corollary 1.5. Let $\Phi: \mathbb{C}^{2} \rightarrow \mathbb{C}$ satisfy $\operatorname{Re} \Phi((1-\alpha) \rho i+\alpha, \tau+i \eta) \leq 0$ when $\rho, \tau$, $\eta \in \mathbb{R}, 0 \leq \alpha<1$ and

$$
2 \tau-\alpha(1-\alpha)\left(1-\rho^{2}\right) \leq 0 .
$$

Let $f \in \mathcal{A}$ with $f^{\prime}(z) \neq 0$. If

$$
\operatorname{Re} \Phi\left(1+\frac{z f^{\prime \prime}(z)}{f^{\prime}(z)}, z^{2} S_{f}(z)\right)>0, \quad \text { where } z \in \mathbb{D}
$$


then $f \in K(\alpha)$.

Remark 1.6. The following functions satisfies the condition (1.3) of Corollary 1.5.

(1) $\Phi_{1}(u, v)=2 v-\alpha$,

(2) $\Phi_{2}(u, v)=2 v+u^{2}-\alpha$,

(3) $\Phi_{3}(u, v)=2 v(1-\alpha)-\alpha(u-1)^{2}$.

Corollary 1.7. Let $f \in \mathcal{A}$ with $f^{\prime}(z) \neq 0$. Then each of the following is a sufficient condition for $f$ to be in $K(\alpha)$.

(1) $\operatorname{Re}\left(2 z^{2} S_{f}(z)-\alpha\right)>0$,

(2) $\operatorname{Re}\left(2 z^{2} S_{f}(z)+\left(1+\frac{z f^{\prime \prime}(z)}{f^{\prime}(z)}\right)^{2}-\alpha\right)>0$,

(3) $\operatorname{Re}\left(2(1-\alpha) z^{2} S_{f}(z)-\alpha\left(\frac{z f^{\prime \prime}(z)}{f^{\prime}(z)}\right)^{2}\right)>0$.

The next theorem gives necessary and sufficient conditions for a function $f \in \mathcal{A}$ to be Janowski convex.

Theorem 1.8. Let $f \in \mathcal{A}$. The following statements are equivalent:

(1) $f \in K[A, B]$.

(2) $\left|2 B \bar{z}+\frac{2\left(1-B^{2} r^{2}\right)-\left(1-r^{2}\right)|A+B|}{A-B} \frac{f^{\prime \prime}(z)}{f^{\prime}(z)}\right|^{2} \leq 2\left(2-\left(1-r^{2}\right)|A+B|\right)$.

(3) $\left|1+\frac{z f^{\prime \prime}(z)}{f^{\prime}(z)}-\frac{1-A B r^{2}}{1-B^{2} r^{2}}\right| \leq \frac{(A-B) r}{1-B^{2} r^{2}}$.

(4) $\frac{2\left(1-B^{2} r^{2}\right)\left(1-r^{2}\right)-\left(1-r^{2}\right)^{2}|A+B|}{A-B}\left|S_{f}(z)\right|$ $+\frac{1}{2}\left|2 B \bar{z}+\frac{2\left(1-B^{2} r^{2}\right)-\left(1-r^{2}\right)|A+B|}{A-B} \frac{f^{\prime \prime}(z)}{f^{\prime}(z)}\right|^{2} \leq 2-\left(1-r^{2}\right)|A+B|$, where $|z|=r<1$.

Moreover, the inequalities (3) and (4) are sharp.

Inequalities (3) and (4) gives the following coefficient bounds.

Corollary 1.9. Let $f(z)=z+\sum_{n=2}^{\infty} a_{n} z^{n} \in K[A, B]$. Then

$$
\left|a_{2}\right| \leq \frac{A-B}{2}, \quad\left|a_{3}\right| \leq \frac{1}{6}(A-B)(A-B+1) .
$$

Moreover, the bounds are sharp.

\section{Proofs of main theorems}

We will use the following lemma.

Lemma 2.1. [4] Let $\Omega \subset \mathbb{C}$ and $\Psi: \mathbb{C}^{2} \times \mathbb{D} \rightarrow \mathbb{C}$ satisfy $\Psi(i \rho, \sigma ; z) \notin \Omega$ whenever $z \in \mathbb{D}$, $\rho$ real and $\sigma \leq-\left(1+\rho^{2}\right) / 2$. If $p$ is analytic in $\mathbb{D}$ with $p(0)=1$, and $\Psi\left(p(z), z p^{\prime}(z) ; z\right) \in$ $\Omega$ for $z \in \mathbb{D}$, then $\operatorname{Re} p(z)>0$ in $\mathbb{D}$. 
Proof of Theorem 1.1. Let $p: \mathbb{D} \rightarrow \mathbb{C}$ be defined as

$$
p(z)=\frac{(A-B) f^{\prime}(z)+(1-B) z f^{\prime \prime}(z)}{(A-B) f^{\prime}(z)-(1+B) z f^{\prime \prime}(z)} .
$$

Then $p$ is analytic and $p(0)=1$. Also, a calculation using equation (2.1) shows that

$$
1+\frac{z f^{\prime \prime}(z)}{f^{\prime}(z)}=\frac{(1+A) p(z)+(1-A)}{(1+B) p(z)+(1-B)}
$$

and

$$
z^{2} S_{f}(z)=\frac{(A-B)\left(4 z p^{\prime}(z)-(A+B+2) p^{2}(z)+2(A+B) p(z)+2-B-A\right)}{2((1+B) p(z)+(1-B))^{2}} .
$$

We define a transformation from $\mathbb{C}^{2} \rightarrow \mathbb{C}^{2}$ as

$$
\begin{aligned}
& u=\frac{(1+A) r+(1-A)}{(1+B) r+(1-B)} \\
& v=\frac{(A-B)\left(4 s-(A+B+2) r^{2}+2(A+B) r+2-B-A\right)}{2((1+B) r+(1-B))^{2}} .
\end{aligned}
$$

Let $\Psi(r, s)=\Phi(u, v)$

$=\Phi\left(\frac{(1+A) r+(1-A)}{(1+B) r+(1-B)}, \frac{(A-B)\left(4 s-(A+B+2) r^{2}+2(A+B) r+2-B-A\right)}{2((1+B) r+(1-B))^{2}}\right)$.

Then

$$
\Psi\left(p(z), z p^{\prime}(z)\right)=\Phi\left(1+\frac{z f^{\prime \prime}(z)}{f^{\prime}(z)}, z^{2} S_{f}(z)\right) .
$$

Hence, according to (1.2), we have $\operatorname{Re} \Psi\left(p(z), z p^{\prime}(z)\right)>0$. We will use Lemma 2.1 to prove that $\operatorname{Re} p(z)>0$.

Taking $r=i \rho$ and $s=\sigma$, we obtain

$$
\begin{aligned}
& u=\frac{(1+A) \rho i+(1-A)}{(1+B) \rho i+(1-B)} \\
& v=\frac{(A-B)\left(4 \sigma+(A+B+2) \rho^{2}+2(A+B) \rho i+2-B-A\right)}{2((1+B) \rho i+(1-B))^{2}} .
\end{aligned}
$$

The condition $\sigma \leq-\left(1+\rho^{2}\right) / 2$ is equivalent to

$$
\frac{2 \tau\left((1-B)^{2}+\rho^{2}(1+B)^{2}\right)^{2}-4 \rho^{2}\left(1-B^{2}\right)\left(A^{2}-B^{2}\right)}{(A-B)\left((1-B)^{2}-\rho^{2}(1+B)^{2}\right)}+(A+B)\left(1-\rho^{2}\right) \leq 0,
$$

where $\tau$ is real part of $v$. On simplification, we have

$$
\begin{aligned}
\rho^{4}\left(2 \tau(1+B)^{4}+\left(A^{2}-B^{2}\right)(1+B)^{2}\right)+ & \rho^{2}\left(4 \tau\left(1-B^{2}\right)^{2}-2\left(A^{2}-B^{2}\right)\left(3-B^{2}\right)\right) \\
& +2 \tau(1-B)^{4}+\left(A^{2}-B^{2}\right)(1-B)^{2} \leq 0,
\end{aligned}
$$

which is equivalent to

$2 \tau\left(\rho^{2}(1+B)^{2}+(1-B)^{2}\right)^{2}+\left(A^{2}-B^{2}\right)\left(\rho^{2}(1+B)-(1-B)\right)^{2}-4 \rho^{2}\left(A^{2}-B^{2}\right) \leq 0$. 
Hence $\operatorname{Re} \Phi\left(\frac{(1+A) \rho i+(1-A)}{(1+B) \rho i+(1-B)}, \tau+i \eta\right)=\operatorname{Re} \Phi(u, v) \leq 0$ using (1.1). This gives $\operatorname{Re} \Psi(\rho i, \sigma) \leq 0$ whenever $\sigma \leq-\left(1+\rho^{2}\right) / 2$.

From Lemma 2.1, we get $\operatorname{Re} p(z)>0$ or equivalently

$$
\frac{(A-B) f^{\prime}+(1-B) z f^{\prime \prime}}{(A-B) f^{\prime}-(1+B) z f^{\prime \prime}} \prec \frac{1+z}{1-z} .
$$

By definition of subordination, there exists an analytic map $w: \mathbb{D} \rightarrow \mathbb{D}$ with $w(0)=0$ and

$$
\frac{(A-B) f^{\prime}+(1-B) z f^{\prime \prime}}{(A-B) f^{\prime}-(1+B) z f^{\prime \prime}}=\frac{1+w(z)}{1-w(z)} .
$$

A simple computation gives

$$
1+\frac{z f^{\prime \prime}(z)}{f^{\prime}(z)}=\frac{1+A w(z)}{1+B w(z)}
$$

and hence

$$
1+\frac{z f^{\prime \prime}(z)}{f^{\prime}(z)} \prec \frac{1+A z}{1+B z}, \quad \text { or } \quad f \in K[A, B] .
$$

Proof of Theorem 1.8. Clearly $(1) \Leftrightarrow(3)$. We show that $(1) \Rightarrow(4) \Rightarrow(2) \Rightarrow(1)$.

Let $f \in K[A, B]$. Then there exists an analytic function $w: \mathbb{D} \rightarrow \mathbb{D}$ with $|w(z)| \leq|z|$ such that

$$
1+\frac{z f^{\prime \prime}(z)}{f^{\prime}(z)}=\frac{1+A w(z)}{1+B w(z)}
$$

This gives

$$
\frac{f^{\prime \prime}(z)}{f^{\prime}(z)}=\frac{(A-B) \phi(z)}{1+B z \phi(z)} \quad \text { or } \quad \phi(z)=\frac{f^{\prime \prime}(z) / f^{\prime}(z)}{(A-B)-B z f^{\prime \prime}(z) / f^{\prime}(z)},
$$

where $\phi(z)=w(z) / z$ is analytic and satisfies $|\phi(z)| \leq 1$ in $\mathbb{D}$. A simple computation gives

$$
\phi^{\prime}(z)=\frac{2(A-B) S_{f}(z)+\left(\frac{f^{\prime \prime}(z)}{f^{\prime}(z)}\right)^{2}(A+B)}{2\left((A-B)-B z f^{\prime \prime}(z) / f^{\prime}(z)\right)^{2}} .
$$

But $\left|\phi^{\prime}(z)\right| \leq\left(1-|\phi(z)|^{2}\right) /\left(1-|z|^{2}\right)$ by the invariant form of Schwarz lemma, so we get

$$
\frac{\left(1-|z|^{2}\right)}{2} \frac{\left|2(A-B) S_{f}(z)+\left(\frac{f^{\prime \prime}(z)}{f^{\prime}(z)}\right)^{2}(A+B)\right|}{\left|(A-B)-B z f^{\prime \prime}(z) / f^{\prime}(z)\right|^{2}} \leq 1-\left|\frac{f^{\prime \prime}(z) / f^{\prime}(z)}{(A-B)-B z f^{\prime \prime}(z) / f^{\prime}(z)}\right|^{2} .
$$

This gives

$$
\begin{aligned}
& \left(1-|z|^{2}\right)(A-B)\left|S_{f}(z)\right|-\frac{\left(1-|z|^{2}\right)}{2}\left|\left(\frac{f^{\prime \prime}(z)}{f^{\prime}(z)}\right)^{2}(A+B)\right| \\
& \leq(A-B)^{2}-\left(1-B^{2}|z|^{2}\right)\left|\frac{f^{\prime \prime}(z)}{f^{\prime}(z)}\right|^{2}-2 B(A-B) \operatorname{Re} \frac{z f^{\prime \prime}(z)}{f^{\prime}(z)}
\end{aligned}
$$


After simplification, we have the desired inequality given by (4).

Clearly $(4) \Rightarrow(2)$. We show that $(2) \Rightarrow(1)$. Opening the square in (2) yields

$$
\left(\left(1-B^{2} r^{2}\right)-\frac{\left(1-r^{2}\right)}{2}|A+B|\right)\left|\frac{f^{\prime \prime}(z)}{f^{\prime}(z)}\right|^{2}+2 B(A-B) \operatorname{Re} \frac{z f^{\prime \prime}(z)}{f^{\prime}(z)} \leq(A-B)^{2} \text {. }
$$

Adding and subtracting $\left(1-B^{2}\right) r^{2}\left|\frac{f^{\prime \prime}(z)}{f^{\prime}(z)}\right|^{2}$ in the left hand side of (2.2), we get $\left(1-B^{2}\right) r^{2}\left|\frac{f^{\prime \prime}(z)}{f^{\prime}(z)}\right|^{2}+\frac{\left(1-r^{2}\right)}{2}(2-|A+B|)\left|\frac{f^{\prime \prime}(z)}{f^{\prime}(z)}\right|^{2}+2 B(A-B) \operatorname{Re} \frac{z f^{\prime \prime}(z)}{f^{\prime}(z)} \leq(A-B)^{2}$.

Since $\frac{\left(1-r^{2}\right)}{2}(2-|A+B|)\left|\frac{f^{\prime \prime}(z)}{f^{\prime}(z)}\right|^{2} \geq 0$ for all $z \in \mathbb{D}$, we get

$$
\left(1-B^{2}\right) r^{2}\left|\frac{f^{\prime \prime}(z)}{f^{\prime}(z)}\right|^{2}+2 B(A-B) \operatorname{Re} \frac{z f^{\prime \prime}(z)}{f^{\prime}(z)} \leq(A-B)^{2} .
$$

Now, if $B \neq-1$, the above equation gives

$$
r^{2}\left|\frac{f^{\prime \prime}(z)}{f^{\prime}(z)}\right|^{2}+\frac{2 B(A-B)}{1-B^{2}} \operatorname{Re} \frac{z f^{\prime \prime}(z)}{f^{\prime}(z)} \leq \frac{(A-B)^{2}}{1-B^{2}} .
$$

Upon simplification, we have

$$
\left|1+\frac{z f^{\prime \prime}(z)}{f^{\prime}(z)}-\frac{1-A B}{1-B^{2}}\right| \leq \frac{A-B}{1-B^{2}},
$$

which means $f \in K[A, B]$. For $B=-1$, inequality (2.3) reduces to

$$
-2(A+1) \operatorname{Re} \frac{z f^{\prime \prime}(z)}{f^{\prime}(z)} \leq(A+1)^{2} .
$$

This gives

$$
1+\operatorname{Re} \frac{z f^{\prime \prime}(z)}{f^{\prime}(z)} \geq \frac{1-A}{2}
$$

which means $f \in K[A,-1]$.

To verify the sharpness for inequality (3), let $1+\frac{z f^{\prime \prime}(z)}{f^{\prime}(z)}=\frac{1+A z}{1+B z}$. We show that $\left|\frac{1+A z}{1+B z}-\frac{1-A B r^{2}}{1-B^{2} r^{2}}\right|=\frac{(A-B) r}{1-B^{2} r^{2}}$. Let $w=\frac{1+A z}{1+B z}$. Then

$$
|w|^{2}-\frac{2 \operatorname{Re} w\left(1-A B r^{2}\right)}{1-B^{2} r^{2}}=\frac{A^{2} r^{2}-1}{1-B^{2} r^{2}} .
$$

Adding $\left(\frac{1-A B r^{2}}{1-B^{2} r^{2}}\right)^{2}$ both sides, we have the desired equality.

To verify the sharpness of inequality (4), we substitute

$$
\frac{f^{\prime \prime}(z)}{f^{\prime}(z)}=\frac{A-B}{1+B z} \quad \text { and } \quad S_{f}(z)=\frac{-\left(A^{2}-B^{2}\right)}{2(1+B z)^{2}}
$$


in the left hand side of inequality (4). We show that

$$
\begin{aligned}
& \frac{2\left(1-B^{2} r^{2}\right)\left(1-r^{2}\right)-\left(1-r^{2}\right)^{2}|A+B|}{|1+B z|^{2}}|A+B| \\
& +\left|2 B \bar{z}+\frac{2\left(1-B^{2} r^{2}\right)-\left(1-r^{2}\right)|A+B|}{1+B z}\right|^{2}-4+2\left(1-r^{2}\right)|A+B|=0 .
\end{aligned}
$$

Simplifying the left hand side of equation (2.4), we get

$$
\begin{aligned}
& \frac{4\left(1-B^{2} r^{2}\right)^{2}-2\left(1-B^{2} r^{2}\right)\left(1-r^{2}\right)|A+B|}{|1+B z|^{2}}-4\left(1-B^{2} r^{2}\right)+2\left(1-r^{2}\right)|A+B| \\
& +4 B\left(2\left(1-B^{2} r^{2}\right)-\left(1-r^{2}\right)|A+B|\right) \operatorname{Re}\left(\frac{z}{1+B z}\right), \\
= & \left(2\left(1-B^{2} r^{2}\right)-\left(1-r^{2}\right)|A+B|\right)\left(\frac{2\left(1-B^{2} r^{2}\right)}{|1+B z|^{2}}-2\right)+4 B\left(2\left(1-B^{2} r^{2}\right)\right. \\
& \left.-\left(1-r^{2}\right)|A+B|\right) \operatorname{Re}\left(\frac{z}{1+B z}\right), \\
= & \left(2\left(1-B^{2} r^{2}\right)-\left(1-r^{2}\right)|A+B|\right)\left(\frac{-4 B\left(\operatorname{Re} z+B r^{2}\right)}{|1+B z|^{2}}\right)+4 B\left(2\left(1-B^{2} r^{2}\right)\right. \\
& \left.-\left(1-r^{2}\right)|A+B|\right) \operatorname{Re}\left(\frac{z}{1+B z}\right), \\
= & -4 B\left(2\left(1-B^{2} r^{2}\right)-\left(1-r^{2}\right)|A+B|\right) \operatorname{Re}\left(\frac{z}{1+B z}\right)+4 B\left(2\left(1-B^{2} r^{2}\right)\right. \\
& \left.-\left(1-r^{2}\right)|A+B|\right) \operatorname{Re}\left(\frac{z}{1+B z}\right), \\
= & 0 .
\end{aligned}
$$

This completes the proof.

\section{References}

[1] Harmelin, R., Locally convex functions and the Schwarzian derivative, Israel J. Math., 67(1989), no. 3, 367-379.

[2] Janowski, W., Some extremal problems for certain families of analytic functions, Ann. Polon. Math., 28(1973), 297-326.

[3] Miller, S.S., Mocanu, P.T., Second-order differential inequalities in the complex plane, J. Math. Anal. Appl., 65(1978), no. 2, 289-305.

[4] Miller, S.S., Mocanu, P.T., Differential subordinations, Monographs and Textbooks in Pure and Applied Mathematics, 225, Dekker, New York, 2000.

[5] Nehari, Z., The Schwarzian derivative and schlicht functions, Bull. Amer. Math. Soc., 55(1949), 545-551.

[6] Nehari, Z., Some criteria of univalence, Proc. Amer. Math. Soc., 5(1954), 700-704.

[7] Nehari, Z., A property of convex conformal maps, J. Analyse Math., 30(1976), 390-393. 
Nisha Bohra

Department of Mathematics, University of Delhi

Delhi-110 007, India

e-mail: nishib89@gmail.com

V. Ravichandran

Department of Mathematics, University of Delhi

Delhi-110 007, India

e-mail: vravi68@gmail.com 